\title{
El rol de la Formación Inicial Docente en el proceso de construcción del saber pedagógico
}

The role of initial teacher training in the process of building pedagogical knowledge

\section{Volumen 20, Número 1 \\ Enero - Abril \\ pp. 1-23}

\author{
Marcela Suckel Gajardo \\ Guillermo Rodríguez Molina \\ Gonzalo Sáez Núñez \\ Daniela Campos Saavedra
}

Citar este documento según modelo APA

Suckel Gajardo, Marcela; Rodríguez Molina, Guillermo; Sáez Núñez, Gonzalo y Campos Saavedra, Daniela. (2020). El rol de la Formación Inicial Docente en el proceso de construcción del saber pedagógico. Revista Actualidades Investigativas en Educación, 20(1), 1-23. Doi. 10.15517/aie.v20i1.40121 


\title{
El rol de la Formación Inicial Docente en el proceso de construcción del saber pedagógico
}

The role of initial teacher training in the process of building pedagogical knowledge

\author{
Marcela Suckel Gajardo ${ }^{1}$ \\ Guillermo Rodríguez Molina² \\ Gonzalo Sáez Núñez \\ Daniela Campos Saavedra ${ }^{4}$
}

\begin{abstract}
Resumen: El presente ensayo se articula en función del proceso de construcción del saber pedagógico que construyen docentes en formación en el marco de la Formación Inicial Docente (FID). El proceso de construcción del saber se articula como una acción compleja, de elaboración propia y auto organización, vinculado a las trayectorias de vida, la formación práctica y la relación entre la teoría y la práctica asociada a la FID. En este escenario de formación inicial, la reflexión constituye uno de los momentos más importantes del proceso de investigación-acción al permitir indagar en el significado de la realidad estudiada y alcanzar cierta abstracción o teorización. De lo anterior se ha encontrado, aspectos incipientes y diferenciadores en los distintos niveles de formación inicial. Desde la FID, el proceso de construcción de saber es abordado mediante las fases de la Investigación-Acción: diagnóstico, se profundiza en temáticas circunscritas al ámbito social y pedagógico; diseño, se preparan secuencias de actividades de aprendizaje con foco en el estudiantado; implementación, de actividades que vinculan estrechamente el contexto y la cultura estudiantil. Los procesos reflexivos desde la FID, en asociación con la formación práctica del futuro profesorado constituirían un primer acercamiento a las trayectorias educativas del profesorado en formación, y contribuirían a desaprender modos de actuación y razonamiento pedagógico para acceder a espacios de análisis y problematización.
\end{abstract}

Palabras clave: saber docente, formación de docentes, práctica pedagógica

Abstract: This essay is articulated based on the process of construction of pedagogical knowledge that teachers build in training within the framework of Initial Teacher Training (FID). The process of building knowledge is articulated as a complex action, of self-elaboration and self-organization, linked to life trajectories, practical training and the relationship between theory and practice associated with IDF. In this scenario of initial training, reflection is one of the most important moments of the action research process by allowing to investigate the meaning of the reality studied and achieve some abstraction or theorization. From the above, incipient and differentiating aspects have been found in the different levels of initial training. From the IDF, the process of knowledge building is approached through the phases of Research-Action: diagnosis, it is deepened in themes circumscribed to the social and pedagogical field; design, sequences of learning activities with a focus on students are prepared; implementation of activities that closely link the context and student culture. The reflexive processes from the IDF, in association with the practical training of future teachers, would constitute a first approach to the educational trajectories of teachers in training, and would contribute to unlearning ways of acting and pedagogical reasoning to access spaces for analysis and problematization.

Key words: know teacher; teacher training; pedagogical practice

1 Profesora Universidad de Concepción, Departamento de Currículum e Instrucción, Chile. Dirección electrónica: marcelasuckel@udec.cl ORDIC https://orcid.org/0000-0001-8576-3346

${ }^{2}$ Profesor Universidad de Concepción, Departamento de Currículum e Instrucción, Chile. Dirección electrónica: grodriguez@udec.cl ORDIC https://orcid.org/0000-0002-3499-1004

${ }^{3}$ Profesor Universidad de Concepción, Departamento de Currículum e Instrucción, Chile. Dirección electrónica: gsaez@udec.cl ORDIC https://orcid.org/0000-0002-0483-3646

4 Profesora Universidad de Concepción, Departamento de Currículum e Instrucción, Chile. Dirección electrónica: dancampos@udec.cl ORDIC https://orcid.org/0000-0001-7818-6608

Ensayo recibido: 30 de junio, 2019

Enviado a corrección: 25 de setiembre, 2019

Aprobado: 2 de diciembre, 2019

Los contenidos de este artículo están bajo una licencia Creative Commons 


\section{Introducción}

Investigaciones de las últimas dos décadas (Fullan, 2010; Hargreaves y Shirley, 2012; Mourshed, Chijioke y Barber, 2010; Organización para la Cooperación y el Desarrollo Económicos [OCDE], 2016; 2017) han proporcionado conocimientos cada vez más precisos respecto de cómo ciertas condiciones y estrategias pueden mejorar efectivamente los microsistemas educativos del aula. Algunos casos muestran cambios pedagógicos exitosos y a gran escala en América Latina (Colbert y Arboleda, 2016; Rincón-Gallardo, 2016), donde las prácticas en el aula, la construcción del desarrollo profesional (Gess-Newsomeet al., 2017) y la reflexión sistemática y profunda de la práctica (Toom, 2006) permitiría alcanzar mayor comprensión de los procesos pedagógicos y el desarrollo de conocimientos en el profesorado (Husu, Toom y Patrikainen, 2008). En consideración de lo anterior, la evidencia demuestra que la forma más directa, aunque altamente compleja, de mejorar el aprendizaje del estudiantado es cambiar las prácticas del profesorado (Schleicher, 2016).

La formación del profesorado se exige desde varios ámbitos, uno de los últimos hallazgos relativos a la acreditación de programas de Formación Inicial Docente (en adelante FID) señala que: "la articulación de la formación disciplinar y pedagógica, y la formación teórica y práctica, se presentan como debilidades transversales en las resoluciones de acreditación de las carreras" (Comisión Nacional de Acreditación [CNA], 2018, p. 61), problemáticas que se encuentran entre los aspectos subrayados como críticos de la FID.

En este contexto, el último informe de análisis de los sistemas educacionales de la Organización para la Cooperación y el Desarrollo Económico (OCDE) (2017) señala que la formación inicial del profesorado debe estar centrada en competencias pedagógicas y en exigir a las instituciones que lo forman que observen, guíen y supervisen al estudiantado en la práctica en las organizaciones escolares, con el propósito de articular los procesos teóricos de la FID con las dinámicas escolares y profundizar en la trayectoria del profesorado en formación y sus prácticas pedagógicas.

La capacidad de analizar y reflexionar crítica y colectivamente sobre la práctica se transforma en elemento articulador que conduce la actividad profesional docente en torno a un conjunto de actividades abordadas como un saber hacer en el marco de una comunidad educativa. A partir de esto, el conocimiento sobre el saber enseñar se reconoce como tal relevando el protagonismo de las personas docentes en el contexto de una organización que reconoce y estimula su liderazgo pedagógico, y la proyección que este tiene en la comunidad (Ley 20.903, 2016). En este contexto, según la política pública en educación, se espera que 
el profesorado tenga la capacidad de intervenir para liderar procesos educativos y promover la valoración de la actividad profesional, en consideración del manejo del currículum y su apropiación, el logro de aprendizaje del estudiantado y el análisis de las necesidades sociales, institucionales y comunitarias en un contexto inclusivo (Ministerio de Educación [MINEDUC], 2017).

Para atender estos desafíos y demandas, la formación práctica constituye uno de los elementos claves en los procesos de la FID, pues brinda espacios fundamentales para los procesos de aprender a enseñar y para el aprendizaje de la profesión. En este contexto, la formación práctica previene que una excesiva teorización genere una distancia entre los programas de formación y los requerimientos del sistema escolar. Para Marcelo (1999), la generación de oportunidades para aprender a enseñar, asentadas en los centros escolares, orientadas a resolver la dicotomía entre teoría y práctica, reconoce que el trabajo del profesorado tiene un carácter situado y distribuido; es decir, se construye a través de interacciones sociales tanto en el mundo académico (en la universidad) como en el mundo profesional (la escuela).

Existe consenso en la literatura en cuanto a que la formación de la futura persona docente durante el período de práctica o prácticum representa un elemento fundamental y de especial importancia en el marco de su formación profesional inicial, al reconocer el valor de la experiencia práctica (Latorre Medina y Blanco Encomienda, 2011; Mauri, Clarà, Colomina y Onrubia, 2015; Sorensen, 2014; Zabalza, 2011; Zeichner, 2011). Es así como "el prácticum constituye un espacio privilegiado para analizar el conocimiento de y para la enseñanza: para la reflexión sobre qué y cómo conoce el profesorado; cómo y quién construye, sistematiza y difunde el conocimiento a partir de la práctica" (González Sanmamed y Fuentes Abeledo, 2011, p.49).

Para Hirmas y Cortés (2014) el prácticum tiene como propósito integrar al estudiantado en un contexto de aprendizaje situado en campos reales que permiten poner en práctica el rol profesional a desempeñar, con el objetivo de que el profesorado en formación adquiera los conocimientos, las habilidades y las competencias necesarias para el ejercicio profesional a partir de un diálogo entre teoría y práctica.

Para el caso de las prácticas en la FID, no solamente se deben considerar los desafíos anteriormente expuestos, sino también la relevancia que tiene para el profesorado en formación las experiencias previas en el sistema escolar en su rol de estudiantes. En este sentido, la formación práctica se transforma en un espacio para la reflexión pedagógica y 
didáctica, que permite la revisión de creencias sobre la enseñanza y el aprendizaje para reconocer la influencia de los modelos heredados y aplicados al ejercicio docente, en contraste con los modelos teóricos o "proposicionales" (Labra 2011); así como para la elaboración del saber de la práctica (Galaz y Fuentealba, 2008; Labra, 2011; Latorre, 2006).

En este contexto, se debe considerar qué saberes elabora el profesorado en formación en sus espacios de aprendizaje formal y no formal, teniendo en cuenta la difícil mediación de los saberes obtenidos de la experiencia práctica (Gervais y Correa, 2004), y que la construcción del saber pedagógico es posible a partir de la reflexión sistemática en torno a la práctica pedagógica (de Tezanos, 2007). Por consiguiente, se torna fundamental evidenciar la reflexión en la acción (Schön, 1983), donde el estudiantado universitario tome consciencia y comunique los saberes que moviliza en su práctica pedagógica.

Desde la perspectiva de la presente revisión, las prácticas en los centros escolares, mediadas por procesos reflexivos inducidos desde la FID, constituyen un espacio vital para la formación del profesorado, al proyectar su desarrollo profesional desde el conocimiento que emerge de la interacción entre la pedagogía como disciplina y las necesidades y experiencias que surgen de la práctica. Este saber es una noción compleja, pues se conforma de aspectos curriculares, prácticos y reflexivos que se relacionan e interactúan desde la totalidad. En consecuencia, no existe separación o fragmentación, dado que el fenómeno educativo se despliega, operacionalmente, en una relación de carácter sistémico y multidireccional (Mancovsky y Monetti, 2012; Vezub y Alliaud, 2012).

\section{Desarrollo}

En este apartado se exponen orientaciones para el desarrollo de las prácticas en la FID, basadas en los principios de interacción dialéctica entre la escuela y la Universidad, que consideran las historias de vida del profesorado y el desarrollo de ciclos de prácticas a partir de los principios de la Investigación Acción. Uno de los conceptos vitales para situar en el centro de la FID la formación práctica se relaciona con el saber pedagógico entendido como:

los conocimientos, construidos de manera formal e informal por los profesores y profesoras; valores, ideologías, actitudes, prácticas; es decir, creaciones del docente, en un contexto histórico cultural, que son producto de las interacciones personales e institucionales, que evolucionan, se reestructuran, se reconocen y permanecen en la vida del profesorado. (Díaz, 2001, p. 171) 
Esta construcción se da dentro de un corpus de conocimientos articulado por la comprensión de la relación dialógica entre teoría y práctica, pues "el saber pedagógico es sistémico y situado, por lo mismo, (es) más que sus partes e incomprensible a la mirada lineal” (Ibáñez, 2008, p. 4). Según Guerreiro (2017) señala que:

La base de conocimiento pedagógico del profesorado no es estática. La construcción del nuevo conocimiento emerge de la investigación o se comparte a través de comunidades profesionales, y este conocimiento necesita ser alcanzado, procesado, evaluado, y ser transformado en el conocimiento para la práctica. (p. 30)

Se hace necesario puntualizar que la construcción del saber pedagógico es un proceso que se expresa de manera natural, fruto de la dinámica de la acción del profesorado. Por ello, se postula capitalizar este proceso en la FID para sistematizar este conocimiento mediante procesos reflexivos que potencien progresivamente la comprensión de la complejidad de la práctica docente.

La composición del saber pedagógico se determina por la naturaleza de los saberes que lo conforman. De acuerdo con Díaz (2001), se pueden distinguir tres componentes del saber pedagógico:

a) Saber teórico: Se describe como teorías y/o saberes que están legitimados por escritos provenientes de fuentes académicas y que forman parte de la argumentación con la cual el profesorado fundamenta su acción; es decir, este saber es una construcción abstracta legitimada académicamente. Para Díaz (2001), "los colectivos pedagógicos, en parte, se constituyen en agentes legitimadores de este saber, que es aceptado, compartido y reconocido y, que podrá ser preservado a través de producciones escritas, orales o digitales" (p.178). Se ha estudiado y documentado, en la literatura, una especie de desvalorización del saber teórico por parte del profesorado, pues al parecer no le encuentran vinculación directa con la práctica. Esta percepción parte en la formación inicial, donde las cuestiones teóricas no dialogan con elementos de las realidades educativas en las que se inserta el estudiantado en formación. La relevancia de entender la teoría como un diálogo con la práctica dará mayor profundidad y solidez a los saberes que emerjan del proceso de construcción del saber pedagógico.

b) Saber Práctico: Está relacionado con el saber hacer, aquí se distingue una tensión entre aplicar un saber teórico (actitud problematizadora) y una respuesta sin un referente previo, "surgen los saberes experienciales, aplicables y demostrables. Uno de 
los núcleos generadores, de nuevos conocimientos en el profesorado, es la práctica la cual se convierte en una instancia en la construcción del saber" (Díaz, 2001, p.179). La práctica se convierte en un elemento imprescindible en la actuación y formación inicial, no solo por lo que tiene de aplicación, sino como generadora de teoría (Díaz, 2001). A este respecto, Tardif (2004) plantea que "las relaciones de los docentes con los saberes no son nunca unas relaciones estrictamente cognitivas; son relaciones mediadas por el trabajo que les proporciona unos principios para afrontar y solucionar situaciones cotidianas" (p. 14).

c) Saber reflexivo: La reflexión es una condición para el proceso de construcción del saber, puesto que "la reflexión personal y colectiva le permite analizar, reconstruir su práctica e interrogarse sobre su trabajo personal y profesional" (Díaz, 2001, p.179). En esta perspectiva, la reflexión es un proceso de reconstrucción de la propia experiencia mediante dos fenómenos simultáneos: (1) reconstruir las situaciones donde se produce la acción, lo que conduce a que el profesorado redefina la situación problemática donde se encuentra, ya sea en atención a características de la situación antes ignoradas, o reinterpretando y asignando nuevo significado a las características ya conocidas; y (2) reconstruirse a sí mismos como docentes, proceso que conduce a tomar conciencia de las formas en que se estructuran conocimientos, afectos y estrategias de actuación.

En este marco, se identifican tres elementos fundamentales en la construcción del saber pedagógico. El primero de estos elementos es el cognitivo, asociado a los procesos donde se genera este saber. El segundo elemento se relaciona con el plano afectivo, asociado a las concepciones de mundo y opciones ideológicas que serán determinantes en su actuar pedagógico. El tercer elemento se relaciona con el carácter procesual del saber pedagógico, asociado a la dinámica de reformulación permanente de los saberes que realiza el profesorado en un contexto histórico y social, definido por la comunidad en la que trabaja (Díaz, 2001).

De modo complementario, estudios realizados acerca de los factores que influyen en el aprendizaje de la enseñanza destacan tres elementos principales: a) factores personales (creencias, capacidades, disposiciones, etc.), b) factores asociados al programa de formación (currículum de formación inicial) y c) factores asociados al campo (prácticas pedagógicas) (González Sanmamed y Fuentes Abeledo, 2011). Entre estos factores, las 
prácticas pedagógicas son consideradas como una oportunidad para vincular en la acción teoría y práctica (Feiman-Nemser, Buchmann y Ball, 1986 en González Sanmamed y Fuentes Abeledo, 2011).

Comprender la dinámica de construcción del saber pedagógico implica necesariamente un análisis de la función que desempeña la formación práctica para el estudiantado en formación en este proceso constructivo. Este análisis requiere comprender la relación que se establece entre la teoría y la práctica asociada a la FID.

Por otra parte, investigaciones que abordan la forma en que el conocimiento de las situaciones de práctica y el conocimiento académico interactúan en la representación que el profesorado elabora de esas situaciones dan cuenta de la dificultad de los y las docentes para establecer relaciones entre el conocimiento teórico y el conocimiento de la práctica, esto debido a la naturaleza de ambos tipos de conocimiento y a cómo dicha vinculación se establece en la elaboración de esa representación (Martínez Gorrotxategi et al., 2016).

Los avances empíricos respecto de la relación teoría y práctica se han centrado en la necesidad de comprender y transformar el pensamiento y la acción, donde la investigación se basa en las problemáticas levantadas desde los contextos de práctica a partir del trabajo colaborativo para teorizar, estudiar y actuar sobre dichas problemáticas, todo en perspectiva de optimizar las oportunidades de aprendizaje del estudiantado y las comunidades (Álvarez Álvarez, 2012; Cochran-Smith y Lytle, 2009).

\subsection{Argumentos para la discusión}

Montecinos y Walker (2011) señalan que la desconexión entre la teoría y la práctica se expresa en una disociación que se observa en dos frentes: por una parte, las instituciones que forman al profesorado tienen la idea que la teoría se aprende primero en la universidad y luego se pone en juego en los centros educativos (Korthagen y Kessels, 2009; Hutchinson, 1997), y por otra, el profesorado tiende a pensar que la práctica es la que enseña a hacer frente a las problemáticas reales de las escuelas y que lo aprendido en la universidad no sirve de mucho para abordarlas (Grossman y Loeb, 2008).

Las principales causas de la ruptura teoría-práctica se manifiestan, entre otras, por el escaso contacto del profesorado con el conocimiento pedagógico disponible y su resistencia a realizar cambios en su forma de pensar, la distancia de la academia respecto a los problemas escolares reales y el lenguaje altamente formalizado que utilizan, la cantidad de requerimientos que guían la acción del profesorado, la complejidad de la enseñanza y el 
desarrollo profesional como objeto de estudio, la ambigüedad de la práctica y su escasa valoración social, la dificultad del profesorado para expresar en palabras su acción didáctica cotidiana, las múltiples teorías sobre la enseñanza formuladas desde diferentes perspectivas y la falta de contacto y colaboración cotidiana entre profesorado y académicos-académicas (Álvarez Álvarez, 2012; Korthagen, 2007; Korthagen y Kessels, 2009). Al respecto, experiencias de trabajo colegiado entre pares de docentes en formación, profesorado del centro educativo y académicos y académicas de la universidad, intencionados desde la FID como metodología de trabajo, han constatado la posibilidad del aprendizaje situado y experiencial, a partir del ejercicio dialógico y el intercambio de opiniones en el centro como en las instancias de reflexión y análisis respecto de la escuela, la labor como docente en formación, el trabajo colaborativo y el fortalecimiento de sus roles en el aula (Sáez, Campos, Suckel y Rodríguez, 2019).

En el ámbito de los procesos de aprendizaje en el aula escolar, se ha evidenciado que el profesorado en formación logra problematizar con respecto a las experiencias de práctica y reflexionar en relación con los niveles de logro del aprendizaje de los niños y las niñas del aula cuando son modelados desde la FID con base metodológica en la Investigación-acción (Sáez, Campos, Suckel y Rodríguez, 2019). A partir de este estudio, se identifican focos analíticos en torno a los procesos de aprendizaje en el aula escolar, centrados en las habilidades cognitivas de los niños y las niñas del aula escolar, y a su accionar docente asociado a su desarrollo y progresión. Este análisis se ve potenciado cuando se realiza desde la estrategia de la colaboración, en específico, entre docentes en formación inicial y sus pares más aventajados (de cursos superiores) (Sáez et al., 2019).

Para el re-establecimiento de la relación entre la teoría y práctica, Korthagen (2010) se centra principalmente en la formulación de su "enfoque realista", que se caracteriza por una continua interrelación de teoría y práctica (Korthagen, 2007, 2010). Las características principales de este modelo son: a) trabajar sobre la base de situaciones reales surgidas durante la formación y que han suscitado inquietudes en el futuro profesorado; b) reflexión por parte de la interacción entre el futuro profesorado e c) intervención guiada. Además, para Álvarez Álvarez (2012):

El énfasis de este modelo de relación teoría-práctica reside en la formación del profesorado y en la capacidad de éste de afrontar reflexivamente sus procesos de enseñanza-aprendizaje. Para ello, el docente debe seguir cinco pasos: (1) actuar como docente, (2) someter sus acciones a reflexión y análisis, (3) tomar conciencia de la 
acción, (4) incorporar cambios en la misma y (5) emitir un juicio sobre la acción nueva y tomar decisiones para la puesta en marcha de un nuevo ciclo. (p. 389)

Desde esta perspectiva de la formación inicial docente y la necesaria vinculación entre la teoría-práctica, emerge la Investigación-Acción (IA) como un paradigma que permite articular las prácticas en la FID para propiciar procesos reflexivos que coadyuven a consolidar el saber pedagógico construido, con tal de responder a los desafíos que emergen desde los contextos educativos.

Elliott (1990) define la investigación-acción como "un estudio de una situación social con el fin de mejorar la calidad de la acción centro de la misma" (p. 88). Para el desarrollo de estos procesos, postula como elemento central la reflexión de la situación humana-social del profesorado, y destaca la formulación de diagnósticos de situaciones iniciales para resolver problemas de la práctica docente. En esta primera fase es fundamental describir -mediante diferentes instrumentos - la problemática que será abordada. Para la fase de la intervención se debe tener como referente la modificación de la situación inicial, desarrollando una propuesta formulada sobre la base de reflexiones teórico-prácticas, este ejercicio traerá una comprensión más profunda de las problemáticas educativas situadas en contextos particulares (Elliott, 1990).

Por su parte, Kemmis (1993) concibe el proceso de investigación como una serie de espirales reflexivas en las que se desarrolla un análisis inicial con base en un estudio exhaustivo de los elementos educativos, sociales y políticos que se relacionan directamente con la situación que se intervendrá.

A partir de los principios expuestos de la IA, se han desarrollado en una universidad chilena procesos formativos en la FID que tienen como eje articulador la formación práctica de las futuras personas docentes. Dicha formación incluye prácticas progresivas a lo largo de su plan de estudio, desde primer a quinto año, las que tiene un carácter incremental y gradual en torno a las tareas y procesos que realiza la persona docente en formación en el campo educativo. El marco metodológico, a partir del cual se despliega la mediación de este proceso de práctica, considera las fases diagnóstico, diseño, implementación, análisis y reflexión. En este contexto, el profesorado en formación realiza un diagnóstico de la realidad educativa, la formulación de una problematización pedagógica, el desarrollo de una propuesta didáctica, la implementación de dicha propuesta y la evaluación y análisis de los resultados. Los productos asociados al desarrollo de la IA y al proceso reflexivo asociado 
contempla la producción escrita de los futuros docentes en las diferentes fases desplegadas. De este modo, mediante la escritura, el futuro docente traduce su accionar en actividad intelectual que fundamenta la acción que desarrolla (Quinteros, Torres y Cardona, 2007).

Al tener como referencia las producciones escritas del profesorado en formación, se ha identificado que estos utilizan dos tipos de conceptos: explícitos e implícitos. Para esto reinterpretan lo que observan en el ámbito escolar y lo conceptualizan con apoyo de fuentes y referentes teóricos, lo que les permite transitar interpretativamente de la práctica pedagógica a la construcción del saber pedagógico (Sáez et al., 2019).

Lo anterior se complementa con los aportes de Whitehead y colaboradores, quienes plantean un cambio fundamental en la relación teoría-practica (Whitehead, 2009, Whitehead y McNiff, 2009), pues, a partir de los planteamientos de la investigación-acción (Stenhouse, 1998; Elliot 1990, 2004, 2010), que concibe al profesorado como investigador o investigadora, ha venido a poner en relieve un cambio en la relación enseñanzainvestigación mediante lo que denominan "living theory". Esta se entiende como "una explicación producida por un individuo por la influencia educativa en su propio aprendizaje, y con los demás en el aprendizaje de la formación social en la que vive y trabaja" (Whitehead, 2009, p. 104). Desde esta perspectiva, se cuestiona el enfoque seguido por la mayor parte de la academia, y se propone la construcción de una teoría de la educación real y contextualizada, desarrollada por los propios agentes educativos a nivel individual y en colaboración con otros docentes. En consideración de las conceptualizaciones expuestas, se plantea la orientación de las instituciones formadoras del profesorado hacia la creación de espacios de formación que otorguen relevancia al rol investigador en el marco de la formación práctica. De esta forma se rompe con la dinámica lineal de enseñar cuestiones teóricas desvinculadas de la interacción dialéctica con la práctica pedagógica.

Wellington (1991) afirma que la reflexión involucra al profesorado en un ciclo de pensamiento y acción basado en la experiencia profesional, lo que implica considerar al profesorado como creativo, no como técnico. Para Kemmis (1993), la reflexión es:

Un proceso de transformación de determinado material primitivo de nuestra experiencia (ofrecido desde la historia y la cultura y mediado por las situaciones que vivimos) en determinados productos (pensamientos comprensivos, compromisos, acciones), una transformación afectada por nuestra concreta tarea (nuestro pensamiento y la acción, y las relaciones entre el individuo y la sociedad), utilizando determinados medios de producción (comunicación, toma de decisiones y acción). (p. 148) 
En esta misma línea, se han evidenciado desafíos didácticos que plantea el profesorado en formación, especialmente en los ámbitos del establecimiento de estrategias de aprendizaje colaborativo, situado o estratégico, la diversidad del estudiantado y la habilidad-conocimiento aplicada a niños y a niñas. Por otra parte, la toma de decisiones está definida por su finalidad pedagógica, la cual es declarada por el desarrollo de habilidades superiores del pensamiento (Sáez et al., 2019).

Los conocimientos teóricos que despliega el profesorado en formación están intencionados desde la formación inicial docente universitaria, magisterio o escuelas normales, con énfasis en la "metodología utilizada en el eje práctico basado en las cuatro fases de la investigación-acción y articulado en los programas o cursos del plan de estudio" (Sáez et al., 2019, p. 8). Lo anterior, se articula con las prácticas progresivas (inmersión en la organización escolar) y las temáticas que emergen en el encuentro teórico-práctico de la propuesta de formación.

En la FID y las prácticas, es relevante promover procesos reflexivos que permitan mirar críticamente las realidades escolares en las que se inserta el profesorado en formación, con tal de iniciar ciclos de innovación, así como también marcar diferencias con la enseñanza recibida en el proceso de sus historias escolares personales. Al respecto, el estudiantado que ingresa a las carreras de Pedagogía ya tiene construida una representación de lo que es ser docente (Bobadilla Goldschmidt, Cárdenas Pérez, Dobbs Díaz y Soto Bustamante, 2009), por lo que se torna relevante abordar el concepto de trayectorias, debido a que en el proceso de construcción de saber pedagógico un componente importante es la trayectoria de vida y, particularmente la trayectoria escolar, lo que implica considerar la temporalidad de las experiencias vividas por las personas, sus historias sociales y biográficas (Clandinin y Connelly, 2000; Montes y Sendón, 2006; Terigi, 2014).

Con respecto a las trayectorias educativas, Suckel, Campos, Sáez y Rodríguez (2019) constatan que desde los relatos del profesorado en formación emergen experiencias favorables y desfavorables que aportan elementos en la demarcación de los saberes asociados al quehacer pedagógico. Estas experiencias se analizan y reinterpretan al enfrentarse a escenarios que impliquen movilizar saberes relacionados con la acción práctica. En este contexto, estas experiencias se hacen conscientes y forman parte del proceso reflexivo en torno a la acción práctica, e influyen en la toma de decisiones pedagógicas en el aula. Algunos de los hallazgos más relevantes con respecto a las 
experiencias de trayectoria educativa, que influyen a la construcción de saber y que emergen de las producciones escritas de los profesores en formación, son (Suckel et al., 2019):

a) Las experiencias favorables que aluden a las relaciones interpersonales construidas con sus pares del aula escolar, las cuales se caracterizan por connotar de forma positiva el proceso de interacción social vivenciado con pares en el espacio educativo.

b) Las prácticas docentes que incorporan el componente afectivo en la relación docente estudiante constituyen otro registro de experiencia positiva para los participantes del estudio.

c) Los procedimientos pedagógicos vinculados al uso de estrategias didácticas dan cuenta de experiencias de construcción de conocimiento que incluye y connota favorablemente la dimensión social implicada en el proceso de construcción.

d) La relevancia del desarrollo de clima social de aula como elemento facilitador de procesos de enseñanza aprendizaje emerge en las trayectorias educativas del profesorado en formación con una referencia favorable y colaboradora en el desarrollo intelectual y social de las personas.

e) El propio desempeño académico del profesorado en formación, en el cual resulta relevante la percepción positiva que como estudiantes tienen respecto del apoyo académico recibido, que se vincula directamente con experiencias escolares en las que se manifiestan altas expectativas y desafíos cognitivos.

En contraste, las experiencias desfavorables ligadas a las trayectorias educativas hacen referencia a una evaluación negativa de las prácticas de enseñanza del profesorado mentor, asociadas principalmente a experiencias de violencia y expresadas de diferentes formas como: violencia verbal y física, violencia en la interacción social en el aula, comunicación de bajas expectativas hacia el estudiantado. Con respecto a los episodios de violencia verbal y física, los relatos dan cuenta de la presencia del castigo como forma de interacción social en el aula (Suckel et al., 2019).

Las trayectorias escolares asociadas a las experiencias de aprendizaje del profesorado en formación han presentado experiencias favorables, donde se destacan aquellas experiencias emanadas de las relaciones interpersonales construidas entre los pares. A medida que avanzan en años de formación, las prácticas son relevadas por el estudiantado como experiencias de aprendizaje significativas. 
En lo que respecta, a la construcción de las concepciones sobre el rol docente se han evidenciado diferencias en cuanto a la contribución y/o impacto que tiene la formación inicial docente y las experiencias "trayectorias" (familiares, sociales y escolares) (Suckel et al., 2019). De las experiencias escolares vividas como estudiantes resaltan las trayectorias educativas, donde la condición socioeconómica constituye un factor determinante en la evaluación que realizan de la escuela. A este respecto, se destaca una particularidad esencial en cuanto a que sus declaraciones tienen como punto de partida sus experiencias de inclusión y/o exclusión social de aula. Es aquí donde la escuela, como espacio social, y en especial el profesorado, impactan con sus acciones provistas de su capital cultural y profesional en las experiencias del estudiantado.

En relación con las experiencias vividas como docentes en formación, estos configuran una visión acerca de la docencia a partir de la síntesis elaborada al integrar sus trayectorias escolares y sus experiencias de prácticas progresivas, experimentadas desde el rol de docente en formación (Suckel et al., 2019).En el periodo más reciente de las trayectorias educativas, específicamente durante el proceso de formación inicial asociado al desarrollo de prácticas progresivas en centros educativos, el estudiantado en formación de diferentes cohortes ha reportado experiencias (Suckel et al., 2019) de aprendizaje y de reflexión pedagógica como instancias vinculadas al tratamiento de la teoría y la práctica. Estas prácticas son reconocidas como el espacio de formación que les permite realizar el vínculo entre la teoría y la práctica a partir de la resignificación de estas experiencias, en las que el profesorado en formación adquiere un rol activo en el proceso de construcción del saber pedagógico.

Lo anterior, lleva a reflexionar y a analizar sobre las características que tiene el proceso de construcción de saber pedagógico en la FID (interactúan historias personales y escolares, habilidades cognitivas, saberes teóricos, prácticos y reflexivos) en el marco de las prácticas progresivas en los centros escolares. Esto permite clasificar los tipos de saberes que se generan en este proceso, para identificar cuál es la perspectiva de la teoría y la práctica subyacente a estos y levantar desafíos pedagógico-educativos que emergen de las escuelas a las que asisten, para alejarse de la perspectiva academicista que tiende a referenciar las prácticas desde las Universidades.

En lo que respecta al rol formador de las Universidades, se propone reflexionar sobre las modalidades de prácticas en la FID, con la finalidad de promover y acompañar los 
procesos dialécticos que desarrolla el estudiantado en formación en la interacción con el sistema escolar y su contribución al proceso de construcción del saber pedagógico.

\subsection{Proposición}

Frente a esto, el estudiantado en formación se ve demandado en el desarrollo de competencias profesionales orientadas a investigar, desarrollar y evaluar los procesos de enseñanza-aprendizaje y ser capaces de reflexionar críticamente sobre su propia práctica (Niemi y Jakku-Sihvonen 2011, p. 60), lo que facilitaría la "interacción dinámica entre teoría y práctica. De esta forma el conocimiento que emana de las prácticas es valorado, puesto de relieve y conceptualizado" (Ojanen y Lauriala 2011, p. 101). Estas conceptualizaciones constituyen una base teórica, disponible cuando tengan que asumir rutinas instaladas en la escuela (Koski y Pollari 2011; Ruuskanen 2011).

Los saberes que construyen el estudiantado en formación en el proceso de práctica, son de tal relevancia, que se requiere de procesos de acompañamiento, de las entidades formadoras, que pongan en el centro las experiencias reelaboradas e interpretadas de las situaciones prácticas en sus múltiples dimensiones, por los docentes en formación (Gelfuso y Dennis, 2014, Mauri, Clarà Colomina y Onrubia, 2013 en Martínez Gorrotxategi et al., 2016).

En este escenario de formación práctica que pone de relevancia las reflexiones del profesorado en formación, la escritura de este proceso resulta fundamental. Así, la profesora o el profesor escritor intelectual mira la educación como un proyecto cultural y la entiende como un proyecto en permanente construcción, en donde la escritura está siempre presente (Quinteros et al., 2007).

En el acompañamiento y andamiaje de este proceso reflexivo, resulta fundamental considerar que el profesorado que se inicia en el ejercicio profesional pedagógico se ve abocado a de construir su práctica inicial en busca de un saber hacer más acorde con la realidad de las escuelas y colegios, y con las expectativas y problemáticas que el estudiantado experimenta. La armonización de la teoría pedagógica con la realidad social del grupo de estudiantes dialoga con la construcción del saber pedagógico (Melgarejo-Draper, 2006), donde el profesorado adquiere competencias relacionadas con la reflexión sobre la propia identidad profesional (Niemi y Jakku-Sihvonen 2011). Esto se entiende desde el antecedente que la mayoría del profesorado enfrenta en sus primeros trabajos con una práctica inserta en teorías o discursos pedagógicos abstractos y generales, que se enseñan 
en las instituciones formadoras, poco o nada contextualizados a las condiciones sociales y culturales que viven las comunidades que requieren el servicio (Restrepo, 2004, p. 51).

Para visibilizar las trayectorias de vida del profesorado en formación y la influencia que estas tienen en la práctica pedagógica, se hace necesario propiciar el distanciamiento del sujeto en relación con los roles, a partir de la separación entre estructuras objetivas o posiciones sociales y las experiencias de los actores o formación de identidades (Svampa, 2000 en Montes y Sendón, 2006). De esta forma, se puede avanzar en la FID con la ruptura de ciclos de reproducción de prácticas o de roles respecto de la pedagogía heredada de la experiencia escolar. En el caso de la formación de docentes, el manejo reflexivo de las imágenes sociales implica abordar aspectos de formación inherentes a las trayectorias educativas desde un contexto socio-histórico.

Las historias de vida comunicadas por el estudiantado en formación evidencian su vida particular y social, reflejan ambientes y diferentes contextos en los que se desarrollan particular y profesionalmente (Huchim Aguilar y Reyes Chávez, 2013). De esta forma, las trayectorias constituyen uno de los elementos del contexto sobre el cual se construye el saber pedagógico.

En consideración con los elementos anteriores, el proceso de construcción se articula como una acción compleja, de construcción propia y auto-organización, integrando la subjetividad e intersubjetividad del saber. Por tanto, este se presenta como una elaboración del sujeto que revela una información organizada y sistematizada. Es relevante puntualizar el rol que juegan las trayectorias de vida en el proceso de construcción de saber. Comprender la dinámica de construcción del saber pedagógico implica necesariamente un análisis de la función que desempeña la formación práctica para el profesorado en formación en este proceso constructivo, lo cual requiere comprender la relación que se establece entre la teoría y la práctica asociada a la FID.

\section{Conclusiones}

El marco de actuación docente, en el escenario de práctica pedagógica presentado mediante este trabajo, se circunscribe a un proceso reflexivo intencionado y colectivo en el espacio de formación académica en una universidad chilena, y en lo específico en la formación de profesores y profesoras de educación primaria. Este planteamiento teórico y metodológico, que incorpora prácticas de innovación pedagógica, implica un cambio de 
perspectiva donde el centro de la formación docente parte desde la práctica hacia la teoría, con una visión que implica una continua interrelación entre ambas (Korthagen, 2010).

Respecto del proceso reflexivo intencionado y colectivo, desde la formación académica, las evidencias empíricas y teóricas muestran que las conceptualizaciones elaboradas por las y los profesores en formación mediante su producción escrita, promueve una comprensión multidimensional de la realidad educativa. Respecto de la producción escrita del profesorado en formación, se aprecia el uso de conceptualizaciones provenientes de la psicología y la sociología para describir la realidad educativa. La integración de estas disciplinas facilita la elaboración teórica del profesorado. Además, se constata que la información recolectada en los centros de práctica se proyecta en sus intervenciones para construir, entre otros, un clima de aula adecuado.

La experiencia práctica en la FID, estructurada mediante las fases de la InvestigaciónAcción, constituye un referente teórico y metodológico para fundamentar la producción escrita mediante un proceso cíclico que promueve el perfeccionamiento intelectual del profesorado en formación (Quinteros et al., 2007).

Se pudo constatar que las y los docentes en formación van construyendo su saber pedagógico desde la interacción experiencial de sus prácticas y los fundamentos sobre los cuales desarrollan sus planteamientos y reflexiones. De forma complementaria se requiere de la dimensión social del proceso de construcción del conocimiento, expresado en un saber compartido.

En este escenario de formación inicial docente, la reflexión constituye uno de los momentos más importantes del proceso de investigación-acción al permitir indagar en el significado de la realidad estudiada y alcanzar cierta abstracción o teorización. La rapidez con que trabaja el profesorado y la inmediatez con que son exigidas sus respuestas les obliga a activar un conocimiento implícito, el cual, consolidado, da paso a esquemas y procedimientos mecánicos con los que actúa en el aula.

Los elementos pedagógicos y didácticos incorporados en la formación práctica tienen como objetivo que el profesorado en formación transite hacia la comprensión de la naturaleza teórico-práctica de la pedagogía, donde cobra especial relevancia la formación práctica, el carácter situado, social y distribuido en la construcción del saber docente y en la validación de los saberes, su transformación y adaptación a las realidades del trabajo cotidiano. 
La problematización de la experiencia de formación práctica situada en el aula escolar y compartida en procesos de interacción socioeducativa con los otros actores educativos: profesor guía, estudiantes de primaria, estudiante par y docente acompañante contribuye a que emerjan las tensiones presentes en el sistema escolar, a que se establezcan las diferentes perspectivas de análisis en torno a estas y a que se orienten las reflexiones hacia la búsqueda de nociones integrativas en torno a la práctica educativa.

Específicamente, el profesorado en formación puede transitar desde una singularización de la práctica docente, traducida en anécdotas, conductas y cuestiones básicas del proceso de inserción práctica, hacia la problematización de las situaciones pedagógicas. Además, se observa un desarrollo avanzado de las competencias prácticas; evidenciado en la re-interpretación de aspectos culturales del centro educativo y prácticas escolares, al profundizar y conceptualizar la práctica en situaciones complejas en distintos escenarios. Esto les permite, por una parte, avanzar en su construcción de saber pedagógico para resolver en cierta medida la tensión teoría y práctica, y por otra, promover la capacidad de reflexión crítica sobre su propia actuación (Niemi y Jakku-Sihvonen, 2011), sus habilidades sociales, la construcción colaborativa del aprendizaje, transitando a niveles más complejos.

En lo que respecta a las instituciones escolares en las cuales se desarrollan las prácticas progresivas en la FID, se sostiene que el profesorado en formación, durante su trayecto formativo, requiere de un acompañamiento adecuado realizado por docentes con experiencia en este tipo de situaciones de formación. Por otra parte, es necesario que se incorporen diferentes centros de práctica para acceder a múltiples realidades y salas de clases en las que se vivencie la diversidad de experiencias de aprendizaje. Estos escenarios no constituyen "espacios idealizados", pues en ellos se expresan múltiples problemáticas. De esta forma se aproxima reflexivamente a la comprensión de los diferentes escenarios en los cuales, el profesorado en formación, tendrá que abordar su inserción profesional.

Las trayectorias educativas se componen de experiencias desfavorables en cuanto a la existencia de episodios de interacción pedagógica violenta y la evaluación negativa de las prácticas de enseñanza vivenciadas. Desde la epistemología convergente vigotskiana, este tipo de experiencias constituirían elementos diferenciadores que facilitarían la ruptura de esquemas cognitivos y/o emocionales poco elaborados para dar paso a la construcción de esquemas más integrados y reflexivos acerca de las experiencias de aprendizaje construidas. En consideración de esta aproximación epistemológica, la formación inicial 
docente se enriquece al concebir al estudiantado de pedagogía como sujetos sociales. Al asumir esta perspectiva fenomenológica y de asimilación crítica se puede propiciar procesos formativos que recojan la historia y contexto de las trayectorias educativas del futuro profesorado.

Respecto del análisis crítico de las trayectorias educativas es posible identificar un elemento mediador, el componente vocacional, que permite al estudiantado analizar sus experiencias de formación práctica, ya sea por contraste o por acople con sus experiencias escolares. En este nuevo escenario, se torna relevante considerar la formación desde principios de inclusión social que posicione en el centro a la persona que se forma, al reconocer que las experiencias que porta el profesorado en formación, también incluyen experiencias de exclusión social y educativa.

La reflexión por parte del estudiantado emerge por el trabajo intencionado desde los espacios de aprendizaje en la formación inicial docente, donde construyen sus saberes, la identidad profesional y se configura su rol como futuros docentes a través de un modelamiento pedagógico asociado al proceso reflexivo.

Desde el punto de vista del profesorado en formación, se da una constante tensión entre teoría y práctica, entre sus saberes y discurso. A partir de este planteamiento, se considera que el saber pedagógico constituiría una episteme en el sentido de representar un principio organizador del discurso pedagógico. La construcción del saber desde esta perspectiva permitiría otorgar niveles crecientes de conciencia reflexiva y autonomía personal y profesional al futuro profesorado.

\section{Referencias}

Álvarez Álvarez, Carmen. (2012). La relación teoría-práctica en los procesos de enseñanzaaprendizaje. Educatio Siglo XXI, 30(2), 383-402.

Bobadilla Goldschmidt, Marcela, Cárdenas Pérez, Ana, Dobbs Díaz, Emily y Soto Bustamante, Ana María. (2009). Los rodeos de la práctica: representaciones sobre el saber docente en el discurso de estudiantes de pedagogía. Estudios pedagógicos (Valdivia), 35(1), 239-252.

Clandinin, D. Jean and Connelly, F. Michael. (2000). Narrative inquiry. Experience and Story in Qualitative Research. San Francisco: Jossey-Bass.

Comisión Nacional de Acreditación. (2018). Carreras de pedagogía: análisis de fortalezas y debilidades en el escenario actual. Santiago de Chile: Comisión Nacional de Acreditación. 
Cochran-Smith, Marilyn y Lytle, Susan. (2009). Inquiry as stance: Practitioner research for the next generation. New York: Teachers College Press.

Colbert, Vicky y Arboleda, Jairo. (2016). Gender differences in educational outcomes: study on measures taken and the current situation in Europe. Bruselas: Education, audiovisual and culture executive agency.

de Tezanos Castiñeiras, Araceli. (2007). Formación de profesores: una reflexión y una propuesta. Revista Pensamiento Educativo, 41(2), 57-75.

Díaz Quero, Víctor. (2001). Construcción del saber pedagógico. Sinopsis Educativa, Revista Venezolana de Investigación, 1(2), 13-40.

Elliott Huxtable, John. (1990). La investigación-acción en educación. Madrid: Morata.

Elliott Huxtable, John. (2004). Using research to improve practice: the notion of evidencebased practice. In Christopher Day and Judyth Sachs (eds), International Handbook on the Continuing Professional Development of Teachers (Part 3). Open University Press: Maidenhead.

Elliott Huxtable, John. (2010). El estudio de la enseñanza y el aprendizaje: una forma globalizadora de investigación del profesorado. Revista Interuniversitaria de Formación del Profesorado, 68(24, 2), 201-222.

Fullan, Michael. (2010). All Systems Go: The imperative of Whale System Reform. Toronto: Corwin/Ontario Principals Council, Thousand Oaks.

Galaz, Alberto y Fuentealba, Rodrigo. (2008). La reflexión como recurso para la mejora de las prácticas docentes en servicio: el caso de las Redes Pedagógicas Locales. En José Cornejo y Rodrigo Fuentealba (Eds.), Prácticas reflexivas para la formación profesional docente ¿qué las hace eficaces? (pp. 141-168). Santiago: Ediciones UCSH.

Gelfuso, Andrea y Dennis, Danielle. (2014). Getting reflection off the page: The challenges of developing support structures for pre-service teacher reflection. Teaching and Teacher Education, 38, 1-11.

Gervais, Colette y Correa, Enrique. (2004). Explicitación del saber de experiencia de los profesores en el contexto de las prácticas docentes: un marco conceptual y metodológico. Íkala, revista de lenguaje y cultura, 9(15), 141-167.

Gess-Newsome, Julie; Taylor, Joseph; Carlson, Janet; Gardner, April; Wilson, Christopher y Stuhlsatz, Molly. (2017). Teacher pedagogical content knowledge, practice, and student achievement. International Journal of Science Education, 41(7), 1-20.

González Sanmamed, Mercedes y Fuentes Abeledo, Eduardo. (2011). El Practicum en el aprendizaje de la profesión docente. Revista de Educación, 354(enero-abril), 47-70.

Grossman, Pamela y Loeb, Susanna. (2008). Alternative Routes to Teaching: Mapping the New Landscape of teacher education. Cambridge, MA: Harvard Education Press. 
Guerriero, Sonia (ed.). (2017). Pedagogical Knowledge and the Changing Nature of the Teaching Profession. Paris: OECD Publishing.

Hargreaves, Andy y Shirley, Dennis. (2012). The global Fourth Way: The quest for educational excellence. Toronto: Ontario Principals Council, Corwin.

Hirmas, Carolina y Cortes, Ignacia. (2014). Primer Seminario sobre Formación Práctica Docente. Santiago, Chile: Organización de Estados Iberoamericanos para la Educación de la Ciencia y la Cultura.

Huchim Aguilar, Donaldo y Reyes Chávez, Rafael (2013). La investigación biográficonarrativa, una alternativa para el estudio de los docentes. Revista Actualidades Investigativas en Educación, 13(3), 1-27. Doi: 10.15517/aie.v13i3.12026

Hutchinson, Tom. (1997). Lifelines intermdiate student's book. United States of America: Oxfor University Press.

Husu, Jukka; Toom, Auli y Patrikainen, Sanna. (2008). Guided reflection as a means to demonstrate and develop student teachers' reflective competencies. Reflective Practice, 9(1), 37-51.

Ibáñez Salgado, Nolfa. (2008). Saber Pedagógico y Práctica Docente: Estudio en Aulas de Educación Parvularia y Básica. Informe final de Investigación FONIDE. Santiago de Chile: MINEDUC.

Kemmis, Stephen. (1993). El currículum, más allá de la teoría de la reproducción. Madrid: Morata.

Korthagen, Fred. (2007). The gap between research and practice revisited. Educational research and evaluation, 13(3), 303-310.

Korthagen, Fred. (2010). La práctica, la teoría y la persona en la formación del profesorado. Revista Interuniversitaria de Formación del Profesorado, 68(24, 2), 83-101.

Korthagen, Fred y Kessels, Joss. (2009). Linking theory and practice: changing the pedagogy of teacher education. Educational Researcher, 28(4), 4-17.

Koski, Kirsti y Pollari, Pirjo. (2011). Teacher training schools - the Finnish way of organizing teacher training. En Marita Kontoniemi and Olli-Pekka Salo (eds.), Educating teachers in the PISA paradise. Perspectives on teacher education at a Finnish university (pp. 1320; Vol. 12). Finland: Publications of Jyväskylä Teacher Training school.

Labra Godoy, Pamela. (2011). Construcción de conocimiento profesional docente: el caso de la formación en la práctica (Tesis para optar al Grado Académico de Doctor en Educación). Universidad Academia de Humanismo Cristiano, Santiago de Chile.

Latorre, Marisol. (2006). Nuevas miradas, viejos problemas: las relaciones entre formación inicial y ejercicio profesional docente. Foro Educacional, (10), 41-64. 
Latorre Medina, María José y Blanco Encomienda, Francisco. (2011). El prácticum como espacio de aprendizaje profesional para docentes en formación. REDU-Revista de Docencia Universitaria, 9(2), 35-54.

Ley 20903. Crea el sistema de desarrollo profesional docente y modifica otras normas. (2016). Recuperada de https://www.leychile.cl/Navegar?idNorma=1087343

Mancovsky, Viviana y Monetti, Elda. (julio, 2012). Acerca de "la crítica pedagógica": Una experiencia de reflexión y análisis sobre nuestro saber pedagógico. Biennale internationale de l'éducation, de la formation et des pratiques professionnelles, Paris, Francia.

Martínez Gorrotxategi, Agurtzane; Mauri Majos, Teresa; Colomina Álvarez, Rosa María; Agirre García, Nerea; Clarà, Marc; Bilbatua Pérez, Mariam; Onrubia Goñi, Javier y Lopez de Arana Prado, Elena. (2016). Prácticas de reflexión colaborativa en el análisis de casos reales en el practicum de la formación inicial del profesorado. Revista del Congrés Internacional de Docència Universitària i Innovació (CIDUI), (3), 1-11.

Marcelo, Carlos. (1999). Ponencia presentada en el Panel Fortalecimiento Inicial de Docentes. Santiago de Chile: MINEDUC, DIVESUP.

Mauri, Teresa, Clarà, Marc, Colomina, Rosa y Onrubia, Javier. (2015). Naturaleza de la interacción en procesos de reflexión conjunta sobre situaciones de la práctica por estudiantes de maestro. Papeles de Trabajo sobre Cultura, Educación y Desarrollo Humano, 11(2), 105-109.

Melgarejo-Draper, Javier. (2006). La selección y formación del profesorado: clave para comprender el excelente nivel de competencia lectora de los alumnos finlandeses. Revista de Educación, extraordinario, 273-262.

Ministerio de Educación. (2017). ¿Hacia dónde avanza el sistema educativo en Chile? Análisis de las recomendaciones OCDE contenidas en Evaluaciones de Políticas Nacionales de Educación: Educación en Chile (2004-2016) en el contexto de la Reforma en marcha. Santiago de Chile: Centro de Estudios.

Montes, Nancy y Sendón, María. (2006). Trayectorias educativas de estudiantes de nivel medio. Argentina a comienzos del siglo XXI. Revista Mexicana de Investigación Educativa, 11(29), 381-402.

Montecinos, Carmen, y Walker, Horacio. (2011). La contribución de los centros de práctica a la formación inicial docente: Perspectivas de docentes de aula y docentes directivos (FONDECYT No 1110505). Santiago de Chile: CONICYT.

Mourshed, Mona; Chijioke, Chinezi y Barber, Michael. (2010). How the world's most improved school systems keep getting better. Londres: McKinsey y Company.

Niemi, Hanneli y Jakku-Sihvonen, Ritva. (2011). Una formación del profesorado basada en la investigación. En Ritva Jakku-Sihvonen y Hannele Niemi (eds), Aprender de Finlandia. La apuesta por un profesorado investigador (pp. 53-75). Madrid: Kaleida. 
Organización para la Cooperación y el Desarrollo Económicos. (2016). Education in Colombia, Reviews of national Policies for Education. Paris: OCDE.

Organización para la Cooperación y el Desarrollo Económicos. (2017). Education in Chile, Reviews of National Policies for Education. Paris: OCDE.

Ojanen, Sami y Lauriala, Anneli. (2011). Una supervisión conceptualizadora de las prácticas: Mejorar el desarrollo profesional del profesorado. En Ritva Jakku-Sihvonen y Hannele Niemi (eds), Aprender de Finlandia. La apuesta por un profesorado investigador (pp. 99-117). Madrid: Kaleida.

Quinteros, Josefina; Torres, Flor y Cardona, María. (2007). El maestro escribe su saber y su hacer. Revista Lenguaje, 35(1), 279-300.

Restrepo, Bernardo. (2004). La investigación-acción educativa y la construcción de saber pedagógico. Educación y Educadores, (7), 45-55.

Rincón-Gallardo, Santiago. (2016). Large scale transformation as widespread cultural change in Mexican public schools. Journal of educational Change, 17(4), 411-436.

Ruuskanen, Pekka. (2011). Challenges and prospects for the University of Jyväskylä Teacher Training School. En Marita Kontoniemi and Olli-Pekka Salo (eds.), Educating teachers in the PISA paradise. Perspectives on teacher education at a Finnish university (pp. 2134; Vol. 12). Finland: Publications of Jyväskylä Teacher Training school.

Sáez, Gonzalo; Campos, Daniela; Suckel, Marcela y Rodríguez, Guillermo. (2019). Práctica colegiada en la formación inicial docente y construcción del saber pedagógico. RMIE, 24(82), 811-831.

Schleicher, Andreas. (2016). Teaching excellence through professional learning and policy reform. Lessons from around the world. International summit in the teaching profession. Paris: OCDE.

Schön, Donald. (1983). The reflective practitioner: how professionals think in action. New York: Basic Books.

Sorensen, Peter. (2014). Collaboration, dialogue and expansive learning: the use of paired and multiple placements in the school practicum. Teaching and Teacher Education, 44, 128-137.

Stenhouse, Lawrence. (1998). Investigación y desarrollo del curriculum. Madrid: Morata.

Suckel, Marcela; Campos, Daniela; Sáez, Gonzalo. y Rodríguez, Guillermo. (2019). Trayectorias educativas de profesores de primaria en formación y la construcción de saber pedagógico. Revista de Estudios y Experiencias en Educación, 18(36), 117-133.

Tardif, Maurice. (2004). Los saberes del docente y su desarrollo profesional. Madrid: Narcea. 
Terigi, Flavia. (2014). Trayectorias escolares e inclusión educativa: del enfoque individual al desafío para las políticas educativas. En Avances y desafíos de la educación inclusiva en Iberoamérica (pp. 71-87). Madrid, España: OEl.

Toom, Auli. (2006). Tacit Pedagogical Knowing At the Core of Teacher's Professionality (Tesis doctoral). Faculty of Behavioural Sciences at the. University of Helsinki.

Vezub, Lea y Alliaud, Andrea. (2012). El acompañamiento pedagógico como estrategia de apoyo y desarrollo profesional de los docentes noveles: Aportes conceptuales y operativos para un programa de apoyo a los docentes principiantes de Uruguay. Montevideo: MEC.

Wellington, Brooker. (1991). The promise of reflective practice. Educational Leadership, 48(6), 4-5.

Whitehead, Jack. (2009). Generating living theory and understanding in action research studies. Action research, 7(1), 85-99.

Whitehead, Jack y McNiff, Jean. (2009). Action research. Living theory. California: SAGE.

Zabalza-Beraza, Miguel. (2011). El practicum en la formación universitaria: estado de la cuestión. Revista de Educación, (354), 21-43.

Zeichner, Kenneth. (2011). Rethinking the connections between campus based courses and field experiences in college and university based teacher education. In Jan Millwater, Lisa Ehrich and Denise Beutel (Eds.), Practical Experiences in Professional Education: A transdisciplinary approach (pp. 37-60). Brisbane: Post Pressed. 
Revista indizada en

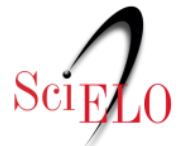

redalyc.sy latindex

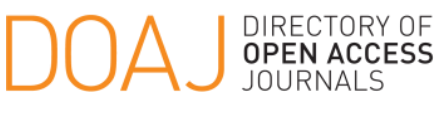

Distribuida en las bases de datos:

- Dialnet

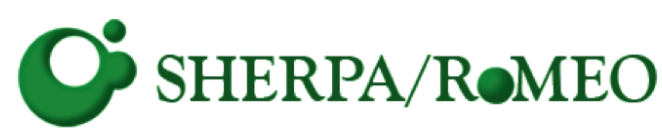

REDIB

Red Iberoamericana

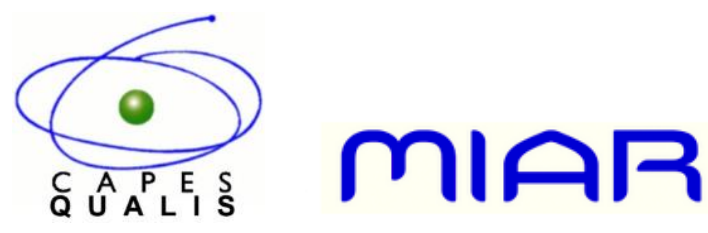

